\title{
Analysis of Digital Hologram Rendering Using a Computational Method
}

\author{
Hyun-Jun Choi ${ }^{1}$, Young-Ho Seo ${ }^{2}$, Seok-Woo Jang ${ }^{3 *}$, and Dong-Wook Kim ${ }^{2}$, Member, KIICE \\ ${ }^{1}$ Department of Electronic Engineering, Mokpo National Maritime University, Mokpo 534-729, Korea \\ ${ }^{2}$ Realistic Media Institute, Kwangwoon University, Seoul 139-701, Korea \\ ${ }^{3}$ Department of Digital Media, Anyang University, Anyang 430-714, Korea
}

\begin{abstract}
To manufacture a real time digital holographic display system capable of being applied to next-generation television, it is important to rapidly generate a digital hologram. In this paper, we analyze digital hologram rendering based on a computer computation scheme. We analyze previous recursive methods to identify regularity between the depth-map image and the digital hologram.
\end{abstract}

Index Terms: Computer-generated hologram, Digital holography, 3D, Holographic television, Hologram

\section{INTRODUCTION}

Active studies on holography, which is the ideal and final goal of 3-dimensional (3D) image display, have been undertaken mainly in the US, Europe, and Japan. In particular, real-time holographic video is the core technology for the next-generation 3D television (TV).

The computer-generated hologram $(\mathrm{CGH})$ was proposed by Brown and Lohmann [1] in 1966. It obtains an interference pattern through an arithmetic operation on a personal computer (PC) by approximating optical signals. Thus, it is easy to obtain a digital hologram (DH) with this CGH method for real and virtual objects. The problem with this method is that it exhausts much calculation time. For example, to calculate a $\mathrm{DH}$ using the $\mathrm{CGH}$ method, approximately 900 seconds are required if a general PC is used to display a significant quantity of computation (3D object measuring approximately $1 \times 1 \times 1 \mathrm{~cm}$ in space). To resolve this problem, Yoshikawa [2] tried to increase the calculation speed by recursively adding only the distance difference between a source point on the 3D object and the digital hologram to be generated. In [3], another recursive technique was proposed where only the leftmost pixel of a row in a digital hologram is fully calculated and the remaining pixels of the row are recursively calculated such as by adding the pre-calculated values and the previously calculated results to the results for the first column pixel of the row.

The purpose of this paper is to analyze a CGH method for calculation speed to generate a digital hologram. We analyze previous recursive methods [3] to identify the regularity between the depth-map image and the digital hologram.

In section II, previous CGH methods are described. Section III contains analysis of the CGH method and then optimization of the CGH method. Our conclusions are given in section IV.

Received 09 December 2011, Revised 15 January 2012, Accepted 25 January 2012

*Corresponding Author E-mail: swjang@anyagn.ac.kr 


\section{COMPUTER-GENERATED HOLOGRAM}

\section{A. Basic Theory of the $\mathrm{DH}$ and CGH}

A system for digital holograms uses electronic equipment such as charge coupled device (CCD) cameras instead of optical ones to record the interference pattern of the holography and transmit it as a video signal. The image is reconstructed on the receiver side by illuminating a laser beam onto the received interference pattern uploaded on a spatial light modulator (SLM). Fig. 1 shows configurations of this system at the transmitter side (a) and the receiver side (b), which are the same configurations as the optical ones other than the CCD camera. That is, the recording system sends laser beams into the collimated wave using the condensing lens, and divides the wave into a reference wave and an object wave using a beam splitter. The object wave is illuminated onto the object while the reference wave is directly illuminated to the CCD camera. Then the two waves form an interference pattern and the CCD camera seizes this pattern. To reconstruct the hologram image, the interference pattern information is uploaded in the SLM to which a collimated wave is illuminated. Then the first diffraction beam is generated and the real image is reconstructed at the same position [4].

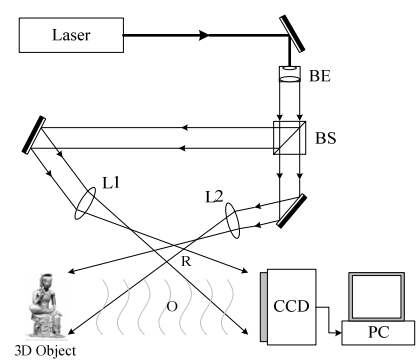

(a)

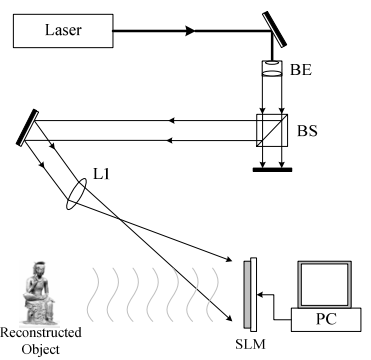

(b)
Fig. 1. Digital hologram; (a) recoding (b) reconstruction.

\section{B. CGH Calculation}

This section describes the previous CGH calculation method and the one using the recursive addition system.

The CGH generating equation is defined as Eq. (1),

$$
I_{\alpha}=\sum_{j}^{N} A_{j} \cos \left(k \sqrt{\left(p x_{\alpha}-p x_{j}\right)^{2}+\left(p y_{\alpha}-p y_{j}\right)^{2}+z_{j}^{2}}\right)
$$

where $\alpha$ or $j$ indicates a particular point on the hologram or $3 \mathrm{D}$ object, respectively, $k$ is the wave number of the reference wave defined as $2 \pi / \lambda, p$ represents the pixel pitch of the hologram, and $\left(x_{\alpha}, y_{\alpha}\right)$ and $\left(x_{j}, y_{j}, z_{j}\right)$ represent the coordinates of the hologram and $3 \mathrm{D}$ object, respectively.
Fig. 2 shows a sample coordinate array system for the 3D object and the digital hologram to apply the CGH method, where the $3 \mathrm{D}$ object in $2 \times 2$, and the DH is captured as $4 \times 4$ in size. To generate a DH with this set-up, the calculation of Eq. (1) must be carried out $64(=2 \times 2 \times 4 \times 4)$ times.

If Eq. (1) is approximated to the first term after the Taylor expansion, it would be as Eq. (2).

$$
\begin{aligned}
I_{\alpha} & =\sum_{j}^{N} A_{j} \cos \left(\frac{2 \pi}{\lambda}\left(z_{j}+\frac{p^{2}}{2 z_{j}}\left(\left(x_{\alpha}-x_{j}\right)^{2}+\left(y_{\alpha}-y_{j}\right)^{2}\right)\right)\right) \\
& =\sum_{j}^{N} A_{j} \cos \left(2 \pi\left(\theta_{z}+\theta_{H}\right)+\phi_{\alpha}+\phi_{j}\right) \\
\left(\theta_{z}\right. & \left.=\frac{z_{j}}{\lambda}, \theta_{H}=\frac{p^{2}}{2 \lambda z_{j}}\left(x_{\alpha j}{ }^{2}+y_{\alpha j}{ }^{2}\right)\right)
\end{aligned}
$$

Here, $x_{\alpha j}$ and $y_{\alpha j}$ mean $\left(x_{\alpha}-x_{j}\right)$ and $\left(y_{\alpha^{-}} y_{j}\right)$. The phase $\theta_{H}\left(x_{\alpha j}+n\right.$, $\left.y_{\alpha j}, z_{j}\right)$ at one point $\left(x_{\alpha}+n, y_{\alpha}\right)$ of the digital hologram can be expressed as,

$$
\begin{aligned}
& \theta_{H}\left(x_{\alpha j}+n, y_{\alpha j}, z_{j}\right) \\
& \left.=\frac{p^{2}}{2 \lambda z_{j}}\left(\left(x_{\alpha j}+n\right)^{2}+y_{\alpha j}{ }^{2}\right)\right)=\frac{p^{2}}{2 \lambda z_{j}}\left(x_{\alpha j}{ }^{2}+y_{\alpha j}{ }^{2}\right)+\frac{p^{2}}{2 \lambda z_{j}}\left(2 n x_{\alpha j}+n^{2}\right) \\
& =\theta_{H}\left(x_{\alpha j}, y_{\alpha j}, z_{j}\right)+\Gamma_{x n}
\end{aligned}
$$

Here, $\Gamma_{x n}$ is defined as,

$$
\Gamma_{x n}=\frac{p^{2}}{2 \lambda z_{j}}\left(2 n x_{\alpha j}+n^{2}\right)
$$

In the case of $\mathrm{n}=1$, that is $\Gamma_{x l}$, Eq. (4) would be,

$$
\Gamma_{x 1}=\frac{p^{2}}{2 \lambda z_{j}}\left(2 x_{\alpha j}+1\right)
$$

Meanwhile, in the case of $\mathrm{n}=2, \Gamma_{x 2}$ is,

$$
\begin{aligned}
\Gamma_{x 2} & =\frac{p^{2}}{2 \lambda z_{j}}\left(4 x_{\alpha j}+4\right) \\
& =\frac{p^{2}}{2 \lambda z_{j}}\left(2 x_{\alpha j}+1\right)+\frac{p^{2}}{2 \lambda z_{j}}\left(2 x_{\alpha j}+1\right)+\frac{p^{2}}{2 \lambda z_{j}} \times 2 \\
& =\Gamma_{x 1}+\Gamma_{x 1}+\Delta_{x}
\end{aligned}
$$

where $\Delta_{x}$ is defined as,

$$
\Delta_{x}=\frac{p^{2}}{2 \lambda z_{j}} \times 2
$$

Again, when $\Gamma_{x 3}$ is calculated with $\mathrm{n}=3$,

$$
\begin{aligned}
\Gamma_{x 3} & =\frac{p^{2}}{2 \lambda z_{j}}\left(6 x_{\alpha j}+9\right) \\
& =\frac{p^{2}}{2 \lambda z_{j}}\left(4 x_{\alpha j}+4\right)+\frac{p^{2}}{2 \lambda z_{j}}\left(2 x_{\alpha j}+1\right)+\frac{p^{2}}{2 \lambda z_{j}} \times 4 \\
& =\Gamma_{x 2}+\Gamma_{x 1}+2 \Delta_{x}
\end{aligned}
$$


When $\mathrm{n}=\mathrm{N}, \Gamma_{x N}$ can be generalized as,

$$
\Gamma_{x N}=\Gamma_{x(N-1)}+\Gamma_{x 1}+(N-1) \Delta_{x}
$$

From Eq. (9) it is clear that, once the first column (the leftmost pixel) of a row of a digital hologram $\left(\Gamma_{x I}\right)$ and $\Delta_{x}$ for a light source are calculated, the remnant pixel values $\left(\Gamma_{x N}\right)$ of the row with the same light source can be recursively calculated by adding $\Gamma_{x l}, \Delta_{x}$, and the previously calculated value $\left(\Gamma_{x(N-1)}\right)$.

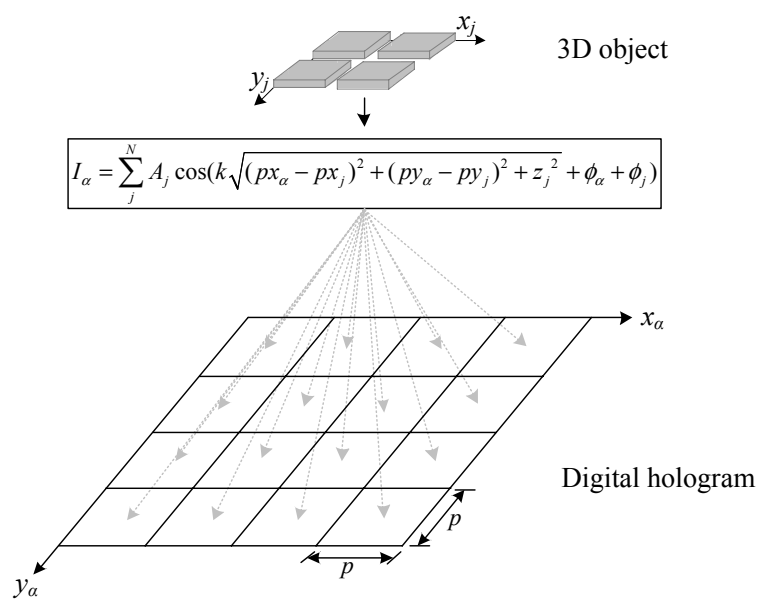

Fig. 2. Computer-generated hologram operation of the conventional method.

At this time, we name the CGH calculation in Eq. (2) full calculation and the calculation by Eq. (9) recursion calculation. Fig. 3 shows the method of [3] described above. The gray blocks in the figure are the results from the full calculations and the white blocks are the results of the recursion calculations. To obtain a $1,024 \times 1,024 \mathrm{DH}$ for an object with the light sources of $200 \times 200$, the ordinary method with only full calculation needs $1,024 \times 1,024 \times 200$ $\times 200=41,943,040,000$ full calculations. But with the previous method, only $1,024 \times 200 \times 200=40,960,000$ full calculations are necessary and the remnant $1,023 \times 1,024 \times$ $200 \times 200=41,902,080,000$ calculations would be recursion calculations. In other words, this method reduces more than $99.9 \%$ of the full calculations and the remnant calculations can be replaced with much simpler recursion calculations.

\section{ANALYSIS OF CGH ALGORITHM}

As discussed in section II-A, the previous CGH method is a more advanced calculation method compared with the conventional method that carries out the full calculation to a level on a par with the quantity of all points of the $3 \mathrm{D}$ object, and the quantity of the coordinates of the hologram.

If the original equation is substituted for $\Gamma_{x l}$ and $\Delta_{x}$ of the Eq. (10), it becomes the same as the following:

$$
\Gamma_{x N}=\frac{p^{2}}{2 \lambda z_{j}}\left(2 x_{\alpha j}+1\right)+\Gamma_{x(N-1)}+\frac{2 p^{2}}{2 \lambda z_{j}}(N-1)
$$

Eq. (11) implies that when one point of the $3 \mathrm{D}$ object is calculated from the first $\mathrm{x}$-axis ( $\mathrm{x}$-axis beginning with the $(0,0)$ coordinate) of the hologram, the variable that changes the value after $\Gamma_{x 2}$ is only $N$. That is, if one point of the $3 \mathrm{D}$ object is computed, the $\Gamma_{x N}$ computed from the first x-axis of the hologram becomes the same as the $\Gamma_{x N}$ values of the other x-axis. Accordingly, the $\Gamma_{x N}$ calculated at the same time with the first $\mathrm{x}$-axis holograms that have been computed can also be used with another x-axis hologram that has been calculated.

The application of the previous method can be expanded to a case where the CGH calculation is carried out on the points that exist on the same column of the 3D object as shown in Fig 4. However, the pre-calculated $\Gamma_{x N}$ values cannot be used repeatedly because, even though the $x_{j}, x_{a}, N$ values are the same when the points on the same column are recursively added using Eq. (11), $z_{j}$ is changed. To solve this, we can process Eq. (11) as below.

In the case of $\mathrm{N}=1, \Gamma_{x l}^{\prime}$ is

$$
\Gamma_{x 1}^{\prime}=\frac{1}{z_{j}}\left(\frac{p^{2}}{2 \lambda}\left(2 x_{\alpha j}+1\right)\right)=\frac{1}{z_{j}}\left(\Gamma_{1}\right)
$$

In the case of $\mathrm{N}=2, \Gamma_{x 2}^{\prime}$ is

$$
\begin{aligned}
\Gamma_{x 2}^{\prime} & =\frac{1}{z_{j}}\left(\frac{p^{2}}{2 \lambda}\left(2 x_{\alpha j}+1\right)\right)+\frac{1}{z_{j}}\left(\frac{p^{2}}{2 \lambda}\left(2 x_{\alpha j}+1\right)\right)+\frac{1}{z_{j}}\left(\frac{2 p^{2}}{2 \lambda}\right) \\
& =\frac{1}{z_{j}}\left(\Gamma_{x 1}+\Gamma_{x 1}+\Delta_{x}\right)
\end{aligned}
$$

In the case of $\mathrm{N}=3, \Gamma_{x 3}^{\prime}$ is

$$
\begin{aligned}
\Gamma_{3 x}^{\prime} & =\frac{1}{z_{j}}\left(\frac{p^{2}}{2 \lambda}\left(2 x_{\alpha j}+1\right)\right)+\frac{1}{z_{j}}\left(\frac{p^{2}}{2 \lambda}\left(2 x_{\alpha j}+1\right)+\frac{p^{2}}{2 \lambda}\left(2 x_{\alpha j}+1\right)+\frac{2 p^{2}}{2 \lambda}\right) \\
& +\frac{1}{z_{j}}\left(\frac{2 p^{2}}{2 \lambda} \times 2\right) \\
& =\frac{1}{z_{j}}\left(\Gamma_{x 1}+\Gamma_{x 2}+2 \Delta_{x}\right)
\end{aligned}
$$

And $\Gamma^{\prime}{ }_{x N}$ in the case of $\mathrm{n}=\mathrm{N}$ can be generalized as follows.

$$
\Gamma_{x N}^{\prime}=\frac{1}{z_{j}}\left(\Gamma_{x(N-1)}+\Gamma_{x 1}+(N-1) \Delta_{x}\right)
$$




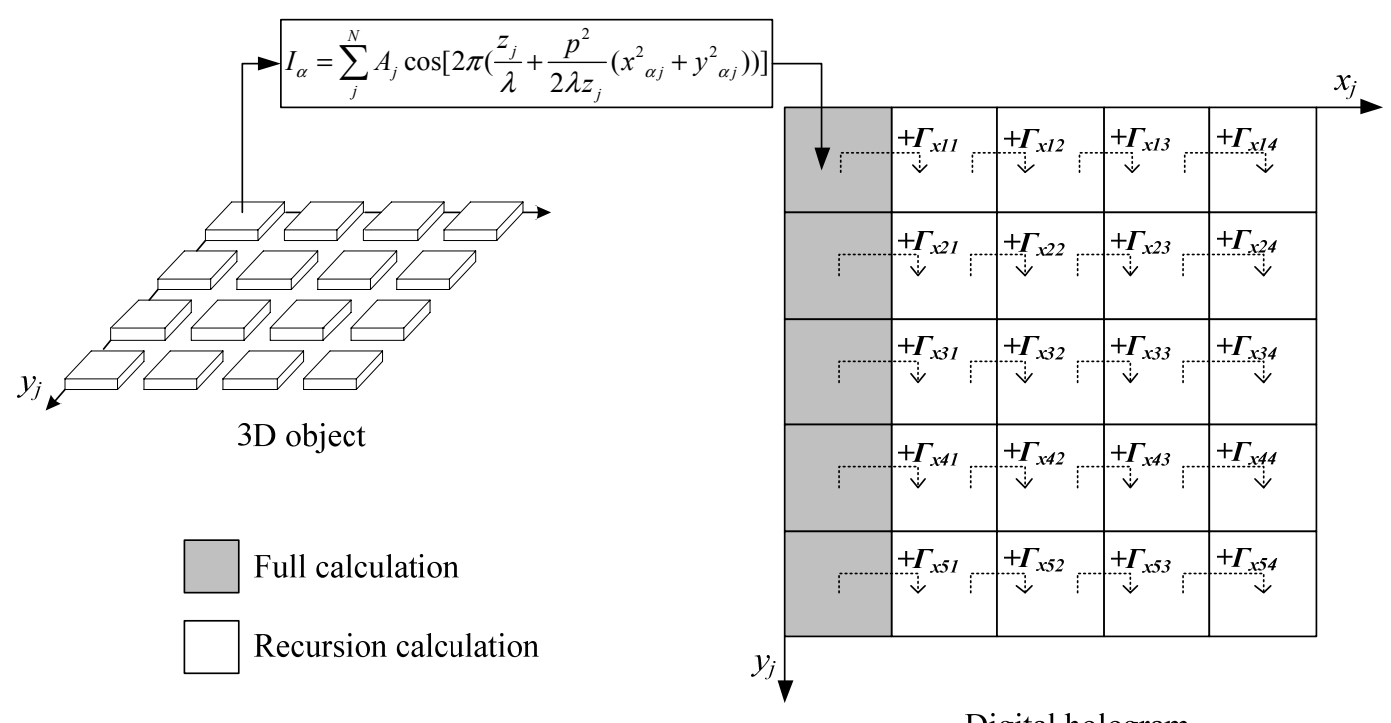

Digital hologram

Fig. 3. computer-generated hologram operation using recursive addition.

As shown in Eqs. (9) and (14), the $\Gamma^{\prime}{ }_{x N}$ values used are identical when carrying out the $\mathrm{CGH}$ calculation on the $3 \mathrm{D}$ objects of the same column, except $z$. This means that when the recursive addition $\mathrm{CGH}$ calculation of the $\mathrm{x}$-axis is carried out on the points that existed in the same column of the $3 \mathrm{D}$ object, the remaining terms, excluding $z_{j}$ can reduce the calculation time by using the $\Gamma_{x N}^{\prime}$ values of the first point as it is.

Applying the $\mathrm{x}$-axis recursive operation method of the previous method explained in section II-B to the $y$-axis direction of the DH can be summarized as follows.

The phase $\theta_{H}\left(x_{a j}, y_{\alpha j}+n, z_{j}\right)$ can be expressed as follows from one point $\left(x_{\alpha}, y_{\alpha}+n\right)$ of the $\mathrm{DH}$.

$$
\begin{aligned}
& \theta_{H}\left(x_{\alpha j}, y_{\alpha j}+n, z_{j}\right) \\
& \left.=\frac{p^{2}}{2 \lambda z_{j}}\left(x_{\alpha j}+\left(y_{\alpha j}+n\right)^{2}\right)\right)=\frac{p^{2}}{2 \lambda z_{j}}\left(x_{\alpha j}{ }^{2}+y_{\alpha j}{ }^{2}\right)+\frac{p^{2}}{2 \lambda z_{j}}\left(2 n y_{\alpha j}+n^{2}\right) \\
& =\theta_{H}\left(x_{\alpha j}, y_{\alpha j}, z_{j}\right)+\Gamma_{y n}
\end{aligned}
$$

Here, $\Gamma_{y n}$ is defined as follows.

$$
\Gamma_{y n}=\frac{p^{2}}{2 \lambda z_{j}}\left(2 n y_{\alpha j}+n^{2}\right)
$$

In the case of $\mathrm{n}=1, \Gamma_{y l}$ is

$$
\Gamma_{y 1}=\frac{p^{2}}{2 \lambda z_{j}}\left(2 y_{\alpha j}+1\right)
$$

and, in the case of $\mathrm{n}=2, \Gamma_{y 2}$ is

$$
\begin{aligned}
\Gamma_{y 2} & =\frac{p^{2}}{2 \lambda z_{j}}\left(4 y_{\alpha j}+4\right) \\
& =\frac{p^{2}}{2 \lambda z_{j}}\left(2 y_{\alpha j}+1\right)+\frac{p^{2}}{2 \lambda z_{j}}\left(2 y_{\alpha j}+1\right)+\frac{p^{2}}{2 \lambda z_{j}} \times 2 \\
& =\Gamma_{y 1}+\Gamma_{y 1}+\Delta_{y}
\end{aligned}
$$

Here, $\Delta_{x}$ is defined as follows:

$$
\Delta_{y}=\frac{p^{2}}{2 \lambda z_{j}} \times 2
$$

Again, when $\Gamma_{y 3}$ is calculated in the case of $\mathrm{n}=3$,

$$
\begin{aligned}
\Gamma_{y 3} & =\frac{p^{2}}{2 \lambda z_{j}}\left(6 y_{\alpha j}+9\right) \\
& =\frac{p^{2}}{2 \lambda z_{j}}\left(4 y_{\alpha j}+4\right)+\frac{p^{2}}{2 \lambda z_{j}}\left(2 y_{\alpha j}+1\right)+\frac{p^{2}}{2 \lambda z_{j}} \times 4 \\
& =\Gamma_{y 2}+\Gamma_{y 1}+2 \Delta_{y}
\end{aligned}
$$

$\Gamma_{y N}$ can be generalized as follows in the case of $\mathrm{n}=\mathrm{N}$.

$$
\Gamma_{y N}=\Gamma_{y(N-1)}+\Gamma_{y 1}+(N-1) \Delta_{y}
$$

When Eqs. (9) and (21) are compared, they are apparently identical. That is, the recursive CGH calculation method in the direction of the x-axis proposed in [3] can be expanded and applied to the y-axis direction.

To find regularities between the points located on the same column of the $3 \mathrm{D}$ object, the actual values were applied to Eq. (21) to calculate $\Gamma_{y N}$, and the results are shown in previous equations. Such regularity can be generalized as Eq. (22). 


$$
\Gamma_{y N}=2 y n+\left(2 y_{\alpha j}+3\right)
$$

Eq. (22) indicates that when the recursive CGH calculation in the direction of the $y$-axis is carried out on the points located in the same column of the $3 \mathrm{D}$ object, the values calculated based on Eq. (22) are recursively added to the value calculated from the first coordinate $((0,0))$ of the hologram. That is, the $\Gamma_{y N}$ that is calculated at the same time with the calculation of the points in the same first column of the $3 \mathrm{D}$ object can be used as it is for the operation on the second points below, reducing the operation time.

\section{CONCLUSIONS}

In this paper, we analyzed a CGH method for calculation speed to generate a digital hologram. We analyzed the previous recursive method [3] to identify the regularity between the depth-map image and the digital hologram.
It is expected that the analyzed $\mathrm{CGH}$ algorithm covering the whole coordinate array of the hologram proposed in this paper will be a core fundamental technology of the holographic 3DTV system for next-generation TV.

\section{REFERENCES}

[1] B. R. Brown and A. W. Lohmann, "Complex spatial filtering with binary masks," Applied Optics, vol. 5, no. 6, pp. 967-969, 1966.

[2] H. Yoshikawa, "Fast computation of Fresnel holograms employing difference," Optical Review, vol. 8, no. 5, pp. 331-335, 2001.

[3] T. Shimobaba and T. Ito, "An efficient computational method suitable for hardware of computer-generated hologram with phase computation by addition," Computer Physics Communications, vol. 138, no. 1, pp. 44-52, 2001.

[4] B. Javidi and F. Okano, Three-Dimensional Television, Video and Display Technology, Berlin, Germany: Springer, 2002.

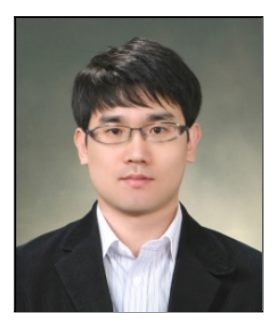

\section{Hyun-Jun Choi}

received his M.S. and Ph.D. degrees in 2005 and 2009, respectively, from the Dept. of Electronic Materials Engineering of Kwangwoon University in Seoul, Korea. He was a research professor in the Realistic Media Institute at Kwangwoon University. He was a professor of the Dept. of Information and Communication Engineering at Anyang University in Anyang, Korea in 2010 to 2011. He is currently an assistant professor with the Department of Electronic Engineering, Mokpo National Maritime University, Mokpo, Korea. He is research interests are in optical image processing and 3D display.

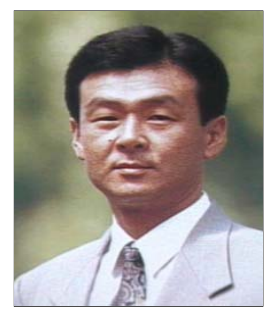

\section{Dong-Wook Kim}

received the B.S. and M.S. degrees from the Department of Electronic Engineering, Hangyang University, Seoul, Korea, in 1983 and 1985, respectively, and the Ph.D. degree from the Department of Electrical Engineering, Georgia Institute of Technology, Atlanta, in 1991. He is currently a Professor and the Dean of Academic Affairs at Kwangwoon University, Seoul. His current research interests include digital system design, digital testability and design-for-test, digital embedded systems for wired and wireless communication, and design of digital signal processors.

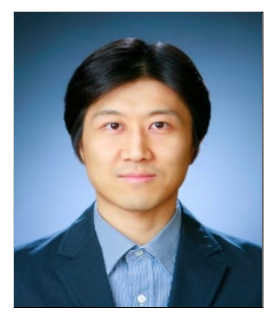

\section{Young-Ho Seo}

received his M.S and Ph.D. degrees in 2000 and 2004, respectively, from Dept. of Electronic Materials Engineering of Kwangwoon University in Seoul, Korea. He was a researcher at Korea Electrotechnology Research Institute (KERI) in 2003 to 2004. He was a research professor in the Dept. of Electronic and Information Engineering at Yuhan College in Buchon, Korea in 2005. He was a professor of the Dept. of Information and Communication Engineering at Hansung University in Seoul, Korea in 2006 to 2007 . He is now an assistant professor of the College of Liberal Arts at Kwangwoon University in Seoul, Korea and a director of a research institute in Ten Technology Inc. His research interests include 2D/3D digital image processing, SoC design, and content security.

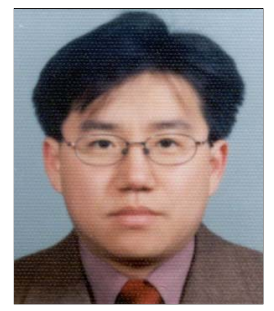

\section{Seok-Woo Jang}

was born in Chunan, Korea, in 1972. He received the B.S., M.S., and Ph.D. degrees in Computer Science from Soongsil University, Seoul, Korea, in 1995, 1997, and 2000, respectively. From October 2003 to January 2009, he was a Senior Researcher in the Construction Information Research Department at Korea Institute of Construction Technology (KICT), Ilsan, Korea. From February 2008 to February 2009, he was a Research Professor in the School of Information and Communication Engineering at Sungkyunkwan University, Suwon, Korea. Since March 2009, he has been an Assistant Professor in the Department of Digital Media, Anyang University, Korea. His primary research interests include robot vision, 3D image processing, augmented reality, biometrics, and pattern recoanition. 